\title{
New views on old memories: re-evaluating the role of the hippocampal complex
}

\author{
R. Shayna Rosenbaum ${ }^{\mathrm{a}, *}$, Gordon Winocur ${ }^{\mathrm{a}, \mathrm{b}, \mathrm{c}}$, Morris Moscovitch ${ }^{\mathrm{a}, \mathrm{c}, \mathrm{d}}$ \\ a Department of Psychology, University of Toronto, 100 St. George St., Toronto, Ont., Canada M5S 1A1 \\ ${ }^{\mathrm{b}}$ Department of Psychology, Otonabee College, Trent University, Peterborough, Ont., Canada K9J $7 B 8$ \\ ${ }^{\mathrm{c}}$ Rotman Research Institute, Baycrest Centre for Geriatric Care, 3560, Bathurst St., Toronto, Ont., Canada M6A $2 E 1$ \\ ${ }^{\mathrm{d}}$ Psychology Department, Baycrest Centre for Geriatric Care, 3560, Bathurst St., Toronto, Ont., Canada M6A 2E1
}

Received 7 April 2001; received in revised form 11 July 2001; accepted 25 July 2001

\begin{abstract}
Evidence of temporally graded retrograde amnesia (RA) following hippocampal damage has fuelled the long-standing belief that memory undergoes a consolidation process, whereby memories are progressively modified in neocortical regions until they are independent of the hippocampal (HPC) complex. Support for this position derives from both the animal and human RA literature, although the results are not consistent. Specifically, consolidation theory does not account for loss of episodic (detail) information in humans and context-dependent information in animals, which often extend back for much of the life span. We discuss an alternative approach, the Multiple Trace Theory, which suggests that the HPC complex contributes to the retrieval of recent and remote episodic and context-dependent memories. According to this view, such memory traces are represented as spatially distributed interactions between the HPC and neocortex that persist for as long as those memories exist. On the other hand, semantic, or context-free, memories can become independent of the HPC as consolidation theory predicts. In support of this view, we report recent accounts of relatively flat RA gradients in autobiographical and spatial detail loss in patients and animal models with extensive bilateral HPC lesions. By comparison, temporally graded RA was observed in tests of semantic and context-free memory. We also report neuroimaging studies in which hippocampal activity, elicited during recollection of autobiographical memories, did not distinguish recent from remote episodes. Our discussion suggests ways to reconcile discrepancies in the literature and guide predictions of the occurrence of flat versus temporally limited gradients of remote episodic and semantic memory loss following lesions to HPC. (C) 2001 Elsevier Science B.V. All rights reserved.
\end{abstract}

Keywords: Hippocampus; Retrograde amnesia; Consolidation; Multiple Trace Theory; Spatial memory; Episodic memory; Semantic memory; Context-dependent memory; Context-free memory

\section{Introduction}

In 1957, Scoville and Milner [66] discovered that bilateral damage to the hippocampal (HPC) complex ${ }^{1}$

\footnotetext{
* Corresponding author. Tel.: +1-416-9787-029; fax: + 1-4169784-811.

E-mail addresses: shayna@psych.utoronto.ca (R.S. Rosenbaum), momos@psych.utoronto.ca (M. Moscovitch).

${ }^{1}$ In humans, except in very rare cases (see [17]), the lesion extends outside the hippocampus proper to structures in the hippocampal complex, which include the hippocampal formation (hippocampus proper, dentate gyrus, and subiculum) and adjacent cortex (entorhinal, perirhinal, and parahippocampal) within the medial temporal lobe (MTL), whereas in animals, the lesion is often restricted to the hippocampal formation. For ease of exposition, we use the abbreviation HPC to refer to the hippocampal complex in humans and hippocampal formation in animals unless noted otherwise.
}

and adjacent regions within the medial temporal lobe (MTL) produces an amnesic syndrome characterized by a profound loss of long-term memory for events occurring subsequent to the damage (anterograde amnesia, AA). Since then, a great deal has been learned about the nature of AA, in particular, that memory loss is not uniform for all types of information. For example, we now know that the loss is most pronounced for information that can be recalled only with conscious awareness as part of explicit or declarative memory [42,75]. Although it is clear that memory for personal experiences is impaired, there is some debate as to whether other types of declarative knowledge such as that which comprises semantic memory, are equally affected [76,84-86]. Memory without awareness (implicit memory), whether perceptual, conceptual, or procedural, is 
relatively well preserved even in profoundly amnesic patients (for reviews, see [65,74]). Likewise, various types of premorbid memories appear to be well retained except for a short period of retrograde amnesia (RA) for events immediately preceding the onset of the lesion (e.g. $[39,40,74])$.

Despite the widely held view that RA is relatively brief and temporally graded, not all evidence is consistent with this pattern $[17,25,44,48]$. A recent revival of interest in remote memory has yielded additional contradictory evidence, which combined with previous findings, has forced investigators to reconsider the state of premorbid memories following HPC damage. In this paper, we examine evidence deriving from human and animal models and argue that the pattern of RA, like that of AA, varies with the type of memory under investigation and probably with the location of the lesion. Specifically, we propose that, to understand the varied pattern of RA following HPC damage, an important distinction must be drawn between semantic and episodic memories in humans and between contextfree and context-dependent memories in animals. Our review suggests that temporally graded RA is observed on tests of semantic and context-free memory, whereas measures of episodic or context-dependent memory are likely to yield extensive memory loss with a flatter gradient. If confirmed, these views challenge traditional consolidation theory and argue in favor of Multiple Trace Theory that, (1) attributes to the HPC formation a unique role in retention and retrieval of episodic, context-dependent memories for as long as they exist; and (2) describes the interaction between HPC and neocortex in forming semantic, context-free memories $[37,44,48]$.

\section{Retrograde amnesia: human studies}

\subsection{Episodic versus semantic memory}

Scoville and Milner's [66] observations of temporally graded RA in the well-studied bitemporal amnesic, HM, were consistent with the prevailing view that remote memories are relatively spared in amnesia. In the following year, similar anecdotal evidence of limited RA was found in two left temporal lobectomy patients [58], one with suspected, and the other with confirmed, damage to the right HPC [57]. Formal testing corroborated these findings in a host of amnesic patients with known or presumed HPC damage following a variety of etiologies $[4,5,60,73,84,89,100]$. Remote memory in such patients was assessed in a variety of tests that measured, for example, memory for autobiographical episodes [26,28], public events and people $[2,10,36,60,72]$, television shows $[68,75]$, as well as other information (e.g. vocabulary [87,89]). The observed pattern of temporally graded RA was interpreted in terms of consolidation theory, which states that a period of time is required to complete those physiological processes needed to form enduring representations (e.g. $[35,37,69-71])$. The HPC is presumed to be necessary for memory retention and retrieval only until consolidation is complete and may even contribute to the process. Afterwards, memories can be retained and recovered without HPC involvement.

One version of this idea states that during consolidation, memories transfer from HPC to neocortex $[8,56,70]$. However, no mechanism has been proposed to explain how a memory is transferred from one structure to another, and there is no evidence that this actually occurs. The more likely account, similar to that proposed by Teyler and DiScenna [81], is that, initially, a memory trace consists of a network of neocortical neurons that is bound to HPC. In the early stages, the HPC simultaneously supports the network and acts as an index through which neocortical information is retrieved. As consolidation proceeds, the neocortical portion of the representation is strengthened, allowing that region to support memory retention and retrieval on its own.

In traditional formulations of consolidation theory, including that of Teyler and DiScenna [81], no distinction is made between different types of memories, whether episodic or semantic, explicit or implicit. However, research in cognitive psychology and neuropsychological investigations of brain-damaged amnesics have shown clearly that memory is not a unitary process. Rather, there are different forms of memory, each governed by different principles, represented in different ways, mediated by different brain structures, and expressed under different conditions [84]. Specifically, an important distinction along these lines is drawn between memory that is explicit (with conscious awareness) and implicit (without conscious awareness). Consistent with this evidence, the AA of patients with MTL damage is selective for specific types of explicit memory, whereas implicit memory is generally spared $[42,75]$.

The question arises as to whether all aspects of explicit memory are equally affected in amnesia. Explicit memory itself can be subdivided further into various components, the chief among them being episodic and semantic memory [83]. Episodic memory refers to conscious recollection of contextually bound personal experiences that occur at a particular time and place. Semantic memory is defined as memory for general knowledge that is context free and includes memory for vocabulary, as well as facts about the world and oneself. 
Building on Tulving's [83] distinction between episodic and semantic memory, Kinsbourne and Wood [25] proposed that episodic, but not semantic, information is lost in amnesia. These investigators used the Galton-Crovitz word-cue technique [12] in which participants are asked to describe an autobiographical episode from their recent or remote past in response to single word cues. Kinsbourne and Wood found that amnesic patients were poor at recalling autobiographical events though they could retrieve generic information pertaining to these words. Kinsbourne and Wood's observations are supported by Vargha-Khadem et al.'s [86] recent discovery that people who had sustained HPC damage at birth or early childhood went on to develop normal semantic memory despite a severely limited capacity for episodic memory. This is not to say that semantic memory is necessarily normal in such patients (see $[18,21])$. It is likely that the HPC is also involved in the acquisition and retention of semantic memories and that damage to this structure renders that process more laborious and less efficient.

The distinction between episodic and semantic memory and their relative vulnerability following MTL lesions has important implications for understanding apparent contradictions in the pattern of deficits observed in RA. As noted earlier, MTL damage does not produce a temporally limited and graded RA in all cases. Recent reviews of the literature on AA [17,48,49] show that autobiographical episodic memory is more severely affected than memory for public events, personalities, and facts about oneself, all of which can be considered aspects of semantic memory. Significantly, it was noted that amnesia for premorbid episodic memories can extend for decades and in some cases encompass a person's entire lifespan, even when lesions were restricted to the HPC complex or the HPC formation. This observation was confirmed in recent studies by Cipilotti et al. [9] (see also Viskontas et al. [88]).

By comparison, deficits in semantic memory were not as severe or as extensive unless the memories had a strong episodic component. Thus, the most severe deficits on tests of public events and personalities were observed when tests included events and personalities that were linked to a particular context and had only brief public exposure. Such events would have had to be experienced at the time of their occurrence, making them more akin to autobiographical episodes. In contrast, those semantic memories that were least affected concerned information that was less contextually and time-dependent, such as facts about oneself and vocabulary. In these cases, even extensive MTL damage produced only a temporally limited RA that typically did not last for more than a few years (see $[9,49,88]$ ). Our view is that amnesia for the most recent memories occurred because they were still contextually bound and not fully integrated into semantic memory [43]. The distinction between contextually bound episodic memory and contextually independent semantic memory is central to our notion of the relationship between the HPC and neocortex in remote memory that we develop later as an alternative to consolidation theory.

\subsection{Spatial memory}

Apart from its role in memory for autobiographical episodes, the HPC is involved in spatial information processing and, indeed, O'Keefe and Nadel [53] argued that the HPC's primary function is in learning allocentric spatial relationships within the environment. The strongest evidence for this view derives from animal research (e.g. [41,52,54]), but a number of studies on patients show that lesions to the right HPC reliably produce deficits in spatial learning and memory. These deficits have been demonstrated in tests involving tabletop environments (e.g. [22,67], as well as tests of largescale outdoor environments (e.g. [30]).

Relatively few studies have investigated remote memory in such patients. It follows that if the HPC is fundamentally involved in spatial processing, anterograde and retrograde memory for spatial information should be equally affected. Available data suggest that this is not the case. Several investigators informally assessed patients' knowledge of spatial environments from their recent and distant pasts by having them sketch floor plans of their homes and navigate through surrounding neighbourhoods. The results showed far greater sparing of remote spatial memories than of recent ones $[4,39,100]$. In general, these reports have been corroborated by more systematic investigation, but with some important qualifications. Teng and Squire [80] and Rosenbaum et al. [62] each tested a single amnesic patient with large bilateral HPC lesions (MRI scans of Rosenbaum et al.'s patient, KC, presented in Fig. 1). Both patients were impaired on tests of new spatial learning, but showed surprisingly preserved spatial memory for neighbourhoods in which they had lived for many years prior to sustaining the lesion, and in one case continues to live. Both patients could estimate direction normally and, in a paradigmatic test of allocentric spatial knowledge [82], determine the correct route from one location to another when the most direct route was inaccessible.

Other evidence suggests that remote spatial memory in these patients is not completely intact. More extensive examination of $\mathrm{KC}$ revealed deficits in identifying detailed features of his environment. This was first evident in his impoverished sketch map with respect to landmark inclusions and his difficulty in identifying specific features (i.e. cities) on outline maps of Ontario and Canada (see Table 1). Rosenbaum et al. [62] presented KC with 48 photographs of landmarks (e.g. school, shopping mall) and salient houses (e.g. next 
door, belonging to friends) taken in his neighborhood, as well as 48 foils, only half of which resembled the target photographs. For each photograph, KC was required to choose a target from the foils and to identify the viewpoint from which the photograph was taken. As part of the latter, he was asked to describe the surrounding environment, which was not visible in the photograph. His performance was compared with that of four controls, two of whom continue to live in $\mathrm{KC}$ 's neighborhood as he did, and two who moved away at the time that $\mathrm{KC}$ sustained his lesion. Unlike all controls who correctly recognized and identified the locations of most of the landmarks and houses, KC recognized only 15 , of which he identified only seven. A more detailed analysis of KC's errors revealed that he did not show the typical preference for similar foils over dissimilar ones, nor was he able to identify the location of any house.

We interpret KC's pattern of impaired and spared remote spatial memory as similar in kind to the general pattern of impaired episodic and preserved semantic memory exhibited by $\mathrm{KC}$, in particular, and amnesics in general. Loss of memory for topographical details and environmental features resembles loss of contextually rich and detailed memories of autobiographical episodes, whether acquired recently or long ago. On the other hand, the bare spatial layout of an environment learned long ago that he did remember, but not of one encountered more recently, represents a schematic cognitive map of the environment and resembled in its essential features the gist of personal experiences or context-independent semantic information that is typically spared in RA.

\subsection{Neuroimaging studies of recent and remote memory}

Converging evidence from neuroimaging studies supports our argument that contextually rich memories, whether for autobiographical episodes or environments, are especially dependent on the MTL, whether the memories are recent or remote. If the HPC were to have relinquished its role to the neocortex as memories age, as suggested by traditional consolidation theory, this would have been expressed as changes in activation across time period, such that the HPC would be less active and the neocortex more so as one recounts older memories relative to newer ones. However, when compared against non-episodic conditions, the HPC, either bilaterally or on the left side, is activated more strongly during recognition, recall, or re-experiencing of autobiographical events, with no relationship to the age of the memory (see [29] for review). For example, Fink et al. [16] contrasted brain regions engaged during personal and non-personal autobiographical retrieval using PET. During scanning, seven healthy young adults were presented with personal episodes from across their lifespan (i.e. childhood, adolescence, early adulthood) and nonpersonal episodes experienced by strangers that participants heard about just once prior to scanning. Comparison of these two conditions allowed for the dissociation of subjective re-evoking of the emotional and contextual details that define personal from nonpersonal episodic experiences. Subtraction of the nonpersonal from the personal conditions yielded MTL activation predominantly in the right hemisphere including the HPC, parahippocampus, and amygdala.
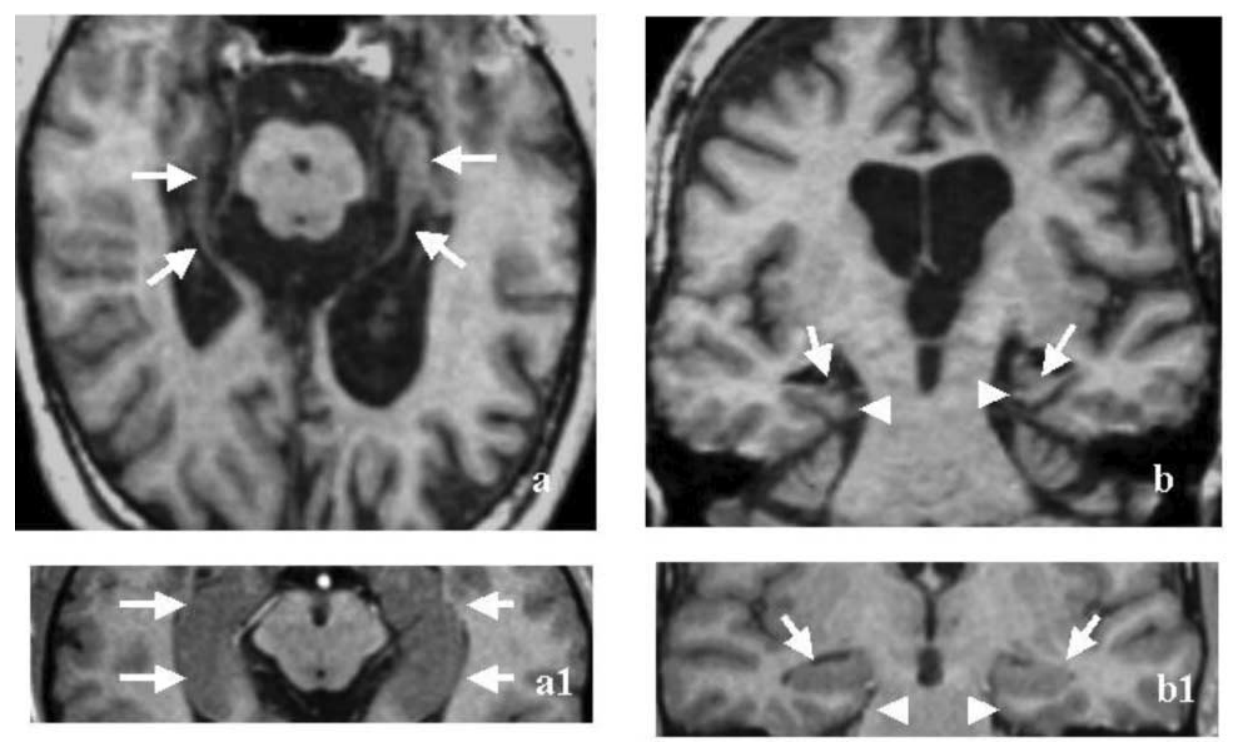

Fig. 1. Magnetic resonance imaging (MRI) slices showing KC's pathology. (a) Axial view showing severely atrophic right and left hippocampus (arrows). (a1) Axial view showing controls's intact right and left hippocampus (arrows). (b) Coronal view showing severe atrophy of the right and left hippocampus (arrows) and atrophy of the right and left parahippocampal gyri (arrow-heads). (b1) Coronal view showing controls's intact right and left hippocampus (arrows) and right and left parahippocampal gyri (arrow-heads). 
Table 1

Scores for $\mathrm{KC}$ and controls on neighbourhood and world geography tests of remote spatial memory

\begin{tabular}{|c|c|c|c|c|c|c|}
\hline Test & & $\mathrm{KC}$ & $\mathrm{RC}$ & PC & JS & SM \\
\hline \multicolumn{7}{|l|}{ Sketch mapping } \\
\hline & Streets & $12 / 16$ & $8 / 16$ & $16 / 16$ & $15 / 16$ & $11 / 16$ \\
\hline & Landmarks & $5 / 14$ & $8 / 14$ & $10 / 14$ & $14 / 14$ & $13 / 14$ \\
\hline \multicolumn{7}{|l|}{ Vector mapping } \\
\hline & Distance & 3.4 & 3.2 & 2.9 & 3.6 & 3.5 \\
\hline & Direction & 11 & $46^{\mathrm{a}}$ & 9 & 13.5 & 7.8 \\
\hline Blocked-route problem solving & & $8 / 8$ & $8 / 8$ & $8 / 8$ & $7 / 8$ & $8 / 8$ \\
\hline Distance judgments & & 0.35 & 0.39 & 0.26 & 0.26 & 0.40 \\
\hline Proximity judgments & & $6 / 6$ & $6 / 6$ & $6 / 6$ & $6 / 6$ & $5 / 6$ \\
\hline Landmark sequencing & & $6 / 7$ & $7 / 7$ & $7 / 7$ & $7 / 7$ & $7 / 7$ \\
\hline \multicolumn{7}{|l|}{ Landmarks and houses } \\
\hline & Recognition & $15 / 48$ & $45 / 48$ & $46 / 48$ & $44 / 48$ & $47 / 48$ \\
\hline & Identification & $7 / 15$ & $43 / 45$ & $46 / 46$ & $43 / 44$ & $37 / 47$ \\
\hline \multicolumn{7}{|l|}{ Map test } \\
\hline & World & $7 / 7$ & $7 / 7$ & $7 / 7$ & $7 / 7$ & $7 / 7$ \\
\hline & North America & $10 / 10$ & $10 / 10$ & $10 / 10$ & $10 / 10$ & $10 / 10$ \\
\hline & Canada & $8 / 18$ & $14 / 18$ & $14 / 18$ & $18 / 18$ & $17 / 18$ \\
\hline & Ontario & $2 / 11$ & $6 / 11$ & $11 / 11$ & $11 / 11$ & $9 / 11$ \\
\hline
\end{tabular}

All tests are scored as number correct with the exception of Vector Mapping-distance and Distance Judgments, which are scored as mean deviation from the correct distance in centimeters and kilometers, respectively, and Vector Mapping — direction, which is scored as mean deviation from the correct direction in degrees.

${ }^{a} \mathrm{RC}$ 's high distance score reflects errors in confusing right with left; the absolute mean deviation of RC's judged directions from the actual directions in degrees is 6 .

In another PET study, Conway et al. [11] had subjects construct a personal memory in response to a visually presented, non-personal word cue, as in the Crovitz technique [12]. Conway et al. [11] reported HPC activity common to both cued-recall of recent and remote autobiographical episodes and of newly acquired paired-associates. Interestingly, a left hemisphere network of activity in frontal cortex was unique to the recent episodic condition. This finding is important in that it would not be predicted on the basis of traditional consolidation theory, which posits that remote, not recent, memories are more dependent on neocortex, while the reverse is true for the HPC.

Similarly, Ryan et al. [63] used fMRI to image seven older adults as they recalled details of 10 autobiographical episodes from each of recent (within the last 2 years) and remote (at least 20 ago) time periods. In addition, subjects participated in a sentence completion condition, which made use of non-autobiographical semantic memory, and a rest condition during scanning. Most striking was the finding of equivalent levels of HPC activity in both the recent and remote task conditions when contrasted with each of the control conditions. Of the seven individuals scanned, five showed significant bilateral HPC activation, one showed significant activation only in the right HPC, and one showed significant activation only in the left HPC. Maguire and Mummery [33] also identified pref- erential activity in the left HPC in addition to activity in medial prefrontal cortex and left temporal pole for retrieval of personally significant, autobiographical episodes that are tied to a particular time period in comparison to memories for public events and personal facts. Moreover, age of memory bore no relation to HPC activity during recall. As in Conway's [11] study, activation differences between recent and remote memories were noted in (ventrolateral) frontal cortex. Subsequent analyses of these data revealed a dynamic network of HPC-parahippocampal interactions that increased in strength with retrieval of autobiographical events relative to other memory types [32]. As well, Maguire and Mummery examined brain activation during tests of semantic memory and the results are consistent with our hypothesis that retrieval of these memories, whether recent or remote, is not mediated by the HPC. Instead, memory for public events and general and personal facts was associated with activation of middle temporal gyrus and temporal pole.

Two studies of remote spatial memory bear on this issue. In one, Maguire and her colleagues [31] examined brain activation in experienced taxi drivers during recall of familiar and complex routes in London, England. Although the memories were old, the HPC, nonetheless, was strongly activated. In another study, Nunn et al. [51] had participants imagine walking along routes in places they had visited recently or long ago. HPC 
activation was observed in comparison to non-spatial conditions for both remote and recent locations.

The results of these studies are consistent with the idea that the HPC mediates aspects of remote spatial memory, but the question remains unresolved as to whether some kinds of well-learned remote spatial memories could exist independently of the HPC. One possibility is that the HPC is activated, but its involvement is not needed for such memories. A related possibility is that intact individuals may conjure up contextually rich representations of their environment while imagining complex routes. Conceivably, it is these rich representations, rather than mental navigation per se, that are associated with HPC activation.

One way to distinguish among the possible interpretations is to scan patients with MTL damage and compare brain activation during tests of spatial memory with that observed in controls. If amnesic patients and controls perform equally well on some tests of spatial memory, but only the controls show HPC activation, that would indicate that the HPC is involved, but is not necessary, for representing schematic cognitive maps of familiar environments. On the other hand, and contrary to our hypothesis, it is possible that a small amount of HPC tissue that survives MTL damage may be sufficient to support schematic, but not rich, cognitive maps of the environment. Under such circumstances, we would expect performance to be associated with the activation of whatever HPC tissue remains viable in the lesioned patient. That such a result is indeed possible is indicated by Maguire et al.'s [34] recent finding that activation of a small amount of surviving HPC tissue was associated with retrieval of episodic memories.

\section{Retrograde amnesia: animal studies}

For many years, research into the behavioural effects of HPC damage in animals focused almost exclusively on new learning and anterograde memory function. While these studies revealed patterns of AA that paralleled those seen in MTL/HPC amnesia, there was virtually no attempt to determine if RA accompanied this effect for pre-operatively experienced events. Undoubtedly, the development of animal models of RA was hampered by the lack of suitable paradigms. In particular, it was difficult to devise tasks that meet two crucial conditions for testing remote memory. First, the test must assess memory for events that can be identified with a specific time period and, second, it must be possible to show that memory for the information degraded normally over a manageable test interval.

In the early 1990s, tests were developed that met these criteria, and investigations of the effects of HPC lesions on remote memory function began to appear.
Several of these studies revealed a pattern of temporally graded RA in which older memories were spared relative to more recently acquired information $[23,24,77,91,99]$. This pattern was very similar to that initially reported for $\mathrm{HM}$ and other amnesic patients with MTL/HPC damage (e.g. $[40,60,66])$ and was generally interpreted as support for traditional consolidation theory.

Animal research has also enabled careful study of the effects of localized damage to the HPC complex, which includes the HPC formation, perirhinal and entorhinal cortex, and parahippocampal gyrus, on remote memory. Although still in its early stages, increasing evidence suggests that circumscribed lesions produce different outcomes, raising the possibility that the various sub-regions mediate different memory-related processes. For example, selective lesions to HPC formation produce variable patterns (related probably to the types of memory under investigation), whereas lesions that target or extend to neighbouring entorhinal cortex more consistently produce RA with a flat gradient (see $[17,46])$. In this paper, we restrict our review to the HPC formation where, we believe, a coherent cross-species theme is beginning to emerge.

One of the first demonstrations of temporally graded RA in rats with lesions to the HPC utilized a food preference test that was originally developed by Galef et al. [19]. In this procedure, a naive subject rat (S) is paired with a demonstrator rat (D) that has recently sampled a particular food. By interacting with $\mathrm{D}, \mathrm{S}$ learns to prefer that food over other foods that have not been experienced in the same way. In normal rats, the memory for an acquired food preference can last 3-4 weeks.

Rats with HPC lesions acquired the preference normally but forgot it at a faster rate than normal rats. In the retrograde memory test, HPC rats exhibited normal memory for the food preference when it was acquired at relatively long intervals (10 days) prior to surgery but almost complete amnesia when it was acquired within $48 \mathrm{~h}$ of surgery. By comparison, in the retrograde test, normal rats exhibited the strongest memories for recently acquired preferences and increased forgetting as the interval between training and surgery increased. The pattern of anterograde and retrograde memory loss seen in the HPC groups appeared to be specific to this brain region in that lesions to other structures yielded quite different patterns on the same tests. Specifically, rats with lesions to the dorso-medial thalamus displayed no loss of pre-operatively acquired memory [90], whereas rats with lesions to frontal cortex displayed a severe RA that extended across all study-test intervals [97].

In another study, Kim and Fanselow [24] compared rats with HPC lesions and control rats on a test of memory for a fear response conditioned to a tone 
(conditional stimulus, CS) and contextual stimuli, at variable intervals before surgery. When tested post-operatively in the same context, but without the CS present, HPC groups exhibited a temporally graded RA, similar to that reported by Winocur and colleagues $[91,96]$. These results are consistent with the pattern of RA predicted by traditional consolidation theory.

When Kim and Fanselow [24] presented the CS in a different context during post-operative testing, there was no evidence of RA, as all groups displayed equally robust fear behaviour. This discrepancy in response to the CS and context, which is not addressed by consolidation theory, has important implications for the theoretical position favoured here and will be discussed later in the section. For the moment, it can be noted that the absence of memory loss in the CS-only condition may relate to Kim and Fanselow's use of a within-groups testing procedure (see [92]). Rats in each training-test interval were administered both the CS-only and context-only tests, with the context-only test always presented first. It is possible that initial testing in the original context primed the conditioned fear response, thereby increasing the probability that it would be available to the HPC groups in subsequent testing. Indeed, evidence from other paradigms suggests that presenting HPC groups with cues that are related to an apparently lost response can facilitate recovery of that response in subsequent testing $[27,38,93,94]$.

In another example, this time involving monkeys, Zola-Morgan and Squire [99] administered a series of different object discrimination tasks at different times before surgery. When tested on the same tasks post-operatively, sham-operated monkeys exhibited the typical forgetting curve, which reflected better recall of recently acquired discriminations relative to those learned earlier. By comparison, HPC-lesion monkeys displayed the opposite pattern - that is, a temporally graded RA in which the discriminations learned at the beginning of the series were remembered better.

It should be noted that temporally graded RA for learned object discriminations has not always been observed following HPC lesions. For example, in a similar study with monkeys, Salmon, Zola-Morgan, and Squire [64] reported RA that extended over the entire testing period without a gradient. In contrast, Mumby et al. [45], in a study involving HPC-damaged rats, found no memory loss for object discriminations acquired between 1 and 13 weeks pre-operatively. Importantly, the same HPC groups, administered tests of spatial memory, showed extensive RA for places learned up to 14 weeks pre-operatively.

A number of factors may account for the variable results in studies involving object discrimination. One important factor is the extent to which extra-HPC structures were affected by the lesion, most notably the perirhinal cortex. In the Salmon et al. [64] study, which involved combined HPC and amygdala lesions, it is likely that the perirhinal cortex was damaged. Recent evidence suggests that damage restricted to perirhinal cortex leads to severe deficits in object discrimination tests of learning and memory, whereas the deficits are much milder following HPC lesions. By comparison, lesions in the Zola-Morgan and Squire [99] study appear to have been restricted to the HPC formation. Involvement of perirhinal cortex is not a factor in Mumby et al. [45] study where lesions were directed at the dorsal HPC of rats. To account for the difference between Mumby et al.'s results and those of Zola-Morgan and Squire, it is necessary to consider species and lesion differences as well as training procedures that may have introduced different elements into the two studies. We return to these points later when we argue for differential effects of context on memory for pre-operatively acquired information following HPC damage and discuss the implications of these effects for consolidation theory.

\subsection{Spatial memory}

Several investigators have studied the effects of HPC lesions on the recovery of spatial memories learned at different times before surgery. This work is of particular interest, given the considerable evidence that animals with HPC lesions are especially vulnerable on tests of spatial learning and memory (e.g. $[41,54,78,79])$. The results of these studies, which assessed spatial memory in the Morris water maze, are generally consistent in revealing severe RA loss following HPC lesions that accompanied an anterograde deficit in post-operative learning of spatial locations. However, in contrast to the temporal gradients observed in the preceding examples, the gradient of retrograde memory loss in these tasks was generally flat when extended over the full range of the training-surgery intervals under investigation $[3,6,45,61]$.

A recently completed study by Winocur, Moscovitch, and Caruana (in preparation) indicates that the flat gradient can also be demonstrated in other spatial tasks. In this study, rats were trained at 3,6 , or 9 months of age to find reward in a particular arm of a cross-shaped maze and then were tested a few weeks after HPC or control surgery. Regardless of time of training, rats with HPC lesions were severely impaired at recalling the learned preference (as measured by their initial choice on the first test trial) as well as in relearning the location of the food reward. Of particular interest was the finding that HPC and control groups exhibited parallel forgetting curves (see Fig. 2).

It appears that, in animal models, HPC lesions produce RA with a temporal gradient for some types of memories but a flat gradient for others. Spatial memory is a good example of the latter. It should be noted that 
the extensive RA seen in spatial memory poses problems for consolidation theory. On the other hand, it has been suggested that the HPC is uniquely involved in spatial memory and that different rules govern its involvement in this type of memory. In their influential cognitive map theory of HPC function, O'Keefe and Nadel [53] argue that the HPC is critically involved in allocentric spatial information processing and that damage to this structure necessarily results in a failure to form new spatial memories as well as recover previously learned ones, regardless of their age. This argument, however, cannot fully account for the results of some human studies. As noted in the previous section, humans with MTL/HPC damage are able to recover old spatial memories, although many detailed aspects of those memories remain inaccessible [62]. We address the apparent anomaly between animal and human studies in our own research, which we report later in the paper.

\subsection{Context-dependent and context-free memory}

The finding that HPC lesions can produce both temporal and flat gradients in RA is not unique to animals but is also seen in MTL amnesic patients. We have argued above that the type of gradient depends in part on whether the memory to be recalled is semantic or episodic. The representation and storage of semantic memories, which are knowledge-based and context-free, are believed to be mediated by extra-HPC structures and are, therefore, spared when the HPC is damaged.
Associated with this view is the idea that in their early stages, even semantic memories are contextually dependent and their retrieval depends on the integrity of the HPC. However, as they become increasingly independent of context, extra-HPC regions are required for their recall. When that occurs, damage to the HPC is not likely to interfere with their recovery. Nonetheless, episodic memories that are closely linked to spatial, temporal, and related contextual cues depend critically on the HPC. As a result, damage to this structure will affect both the formation of new episodic memories (anterograde) as well as retention and recovery of old ones (retrograde).

At this point, it is not clear how the terms semantic and episodic memory can be applied to animals. On the other hand, the notion of context-free and context-dependent memories, which is embedded in the distinction between episodic and semantic memory, is relevant to animal memory. If context-free and context-dependent memories in animals are analogous in some sense to semantic and episodic memories, respectively, in humans, it follows that context-free memories would resist the effects of HPC damage, whereas memories that are contextually bound would be highly vulnerable.

It is important to distinguish between two usages of the term 'context' to clarify what we mean by contextfree and context-dependent memories. Typically, context refers to the complex of cues that provides the background to the event or task in question. The cues themselves can be linked relationally to each other in a spatial way to form a cognitive map, or non-spatially to

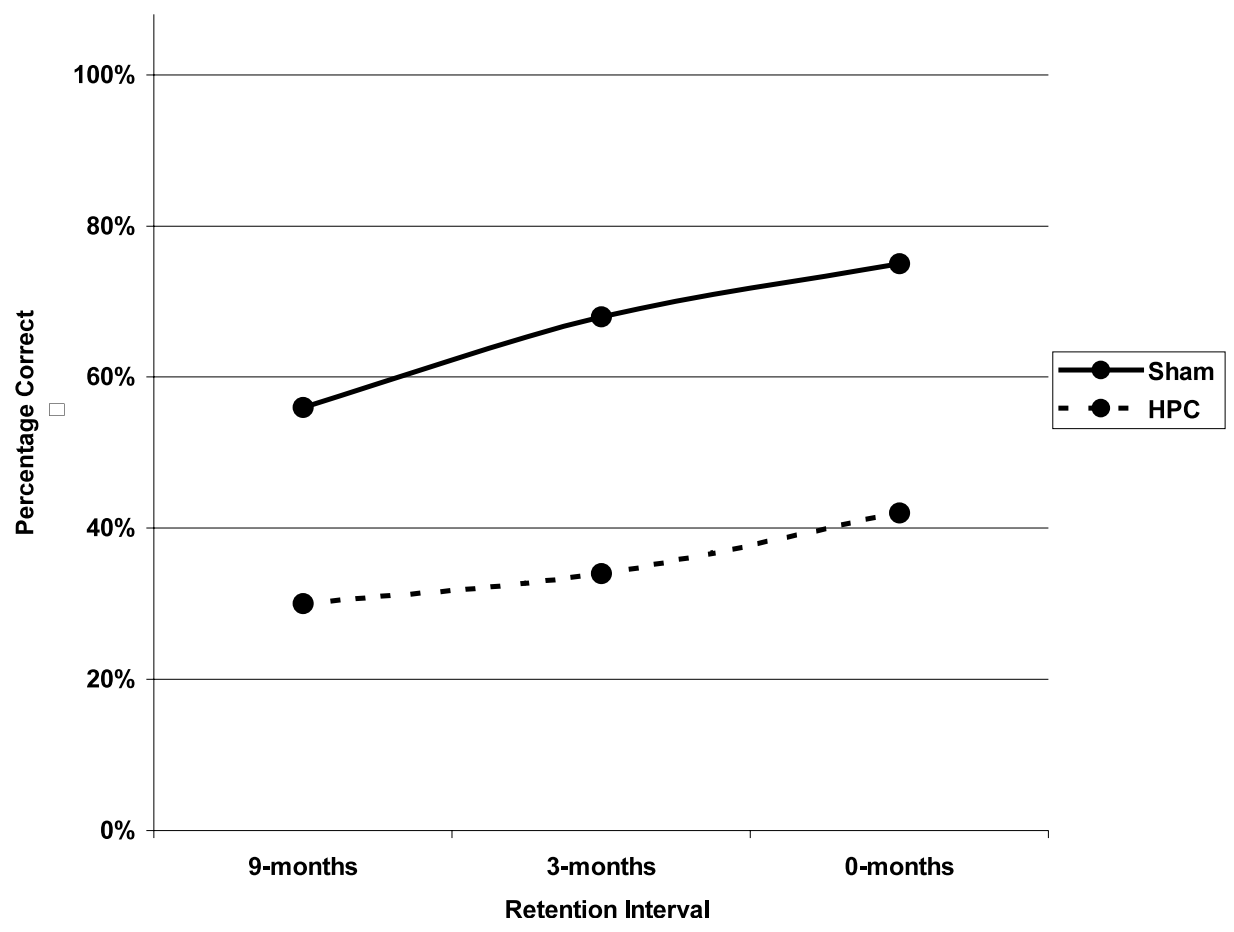

Fig. 2. Memory for a spatial cue preference task in rats following hippocampal or sham lesions. Values represent group means on Day 1 of testing. 
form other types of representations $[13,14]$. In addition, each cue can be linked independently (and non-relationally) to the event that forms the focus of the task at hand. In the latter case, the memory representation consists of simple associations between background cues and elements of the event. The distinction we make between these different ways in which context is represented in memory is similar to the distinction made between relational and associative learning. We therefore will refer to the two types of context as relational and associative. Our position is that HPC lesions selectively affect relational, but not associative, context in anterograde and retrograde memory. A distinguishing feature of our position, not addressed by consolidation theory, is that the extent of remote memory loss following HPC lesions depends on the type of context that is part of the memory trace. In contrast to our view, consolidation theory predicts sparing of all retrograde memories.

An example will illustrate our point. In learning to find a hidden platform in a Morris water maze, the constellation of allocentric cues provides the relational context for locating the platform. Consequently, animals with HPC lesions have difficulty in representing this context and, as a result, are typically impaired on this task. At the same time, each of the cues in the constellation can be linked separately to the platform and provide an associative context for navigation. In such instances, the cues serve as discrete landmarks that signal the location of a platform. Normal animals are not predisposed to use associative context, relying instead on HPC function that enables use of relational context. When the HPC is damaged, animals are restricted to using the landmarks as an associative context to find the platform $[15,47,90,95]$.

We now return to the distinction we drew earlier between context-free and context-dependent memory and relate these terms to our discussion of relational and associative context. By context-dependent, we are referring to relational context, which bears a close resemblance to episodic memory that is defined with respect to a multi-faceted, spatial/temporal context. Context-free memory can refer either to associative context or to knowledge that is represented separately from any context (e.g. semantic memory).

The animal literature on RA following HPC damage is generally consistent with our line of thinking. For example, consider the food preference task, which gives rise to a temporally graded RA in rats with HPC lesions [91]. A normal rat acquires the food preference by interacting with another rat at a particular time and place, in what may be considered a novel context. It is reasonable to infer that, in its initial stage, the memory for the food preference is closely linked to that context in a relational way. However, with the passage of time, the link between the food preference and the relational context becomes less important than the memory for the preference itself, which can be retained without reference to the conditions in which it was acquired. It follows that if the HPC were damaged while the food preference was represented as a contextually bound episodic memory, the representation would be disrupted and the animal's ability to recall the preference post-operatively would be limited. On the other hand, once the preference becomes represented in terms of its essential features and free of contextual elements, it could be recovered without the benefit of the HPC. It is interesting that HPC rats forget the food preference response faster in the anterograde condition, suggesting that when the animal must depend on associative context in one-trial learning, the memory representation is not as durable as when a relational context is used.

Admittedly, this is a somewhat speculative account, but the idea is directly testable. In the standard procedure, food preferences are acquired and recalled in the same compartment and general context. The impact of relational context on memory for food preference can be assessed by administering the test in a totally different environment. If the food preference is initially formed as a context-dependent (relational, episodic) memory and over time becomes a context-free (semantic) memory, no changes are expected in the performance of HPC-lesioned rats. However, the behaviour of normal rats may be affected by changing the context at test when the training-test intervals are short. Changing the context will eliminate many of the cues associated with the food preference. If, at short intervals, the preferences are based on relational context and represented as episodic memories dependent on that context, normal rats might have more difficulty in recovering the preference if the context is absent. Their preference at longer delays should not be affected because, by this time, information about the acquired preference would be represented in a context-free form. On the assumption that HPC-rats do not use contextual cues to form or retrieve the food preference, altering the context should not affect their performance at short or long delays.

The idea that HPC damage selectively affects preoperatively acquired memories that are context-dependent can explain the temporally graded RA for object discrimination, as reported by Zola-Morgan and Squire [99]. As with food-preference learning, we make the assumption that discriminations acquired shortly before surgery are still linked to the relational context and, as a result, are vulnerable to effects of HPC lesions. The Mumby et al. [45] study, which found no RA for object discrimination following HPC lesions, appears to challenge this interpretation, but it is also a challenge to traditional consolidation theory. The notion of contextual linkage in object discrimination, as in food-preference, must be considered a viable hypothesis that 
awaits further experimentation. Systematic variation of context within an object discrimination paradigm should identify the conditions in which context forms part of the memory representation. When those conditions are met, the prediction is that HPC lesions will disrupt a preoperatively acquired memory only if it is dependent on relational context.

The apparently contradictory results regarding HPC lesions and contextual fear conditioning also pose a challenge to understanding the role of the HPC in remote memory. Kim and Fanselow $[1,24]$ report temporally graded RA for a fear response conditioned to context but not to the CS that is part of the stimulus complex being conditioned. However, as indicated above, in their paradigm, context-only conditioning is always tested before CS-only conditioning, so the effect of the prior experience may contaminate the CS-only test. As a result of this confound, one cannot be certain that temporally graded RA is specific to contextual cues. A between-groups design in which different groups are tested in CS-only and context-only conditions would answer this question.

Another concern with respect to contextual fear conditioning is that there is considerable evidence that rats with HPC lesions are impaired in conditioning a fear response to context in the Kim and Fanselow paradigm [24]. As indicated above, this may be because the nature of the environment in that paradigm strongly favours formation of a relational context between a broad array of spatially distributed background cues. When context consists of a limited number of highly salient cues in a restricted environment, the evidence shows that, contrary to Kim and Fanselow's results, rats and other animals with HPC lesions are not impaired in contextual fear conditioning $[20,59,92,98]$. Our view is that, in contrast to the Kim and Fanselow paradigm, where the context is relational, in the other paradigms, the background context is associative. In the latter cases, animals with HPC lesions formed independent associations between the CS, context, and the fear response induced by the unconditional stimulus (US). This was especially clear in the Winocur [92] study. When trained under these conditions and tested in the CS-plus context, CS only, or context only conditions, Winocur found that HPC groups displayed the fear response to equal degrees in all conditions. By comparison, control animals exhibited strong fear conditioning only in the CS-plus context test condition. These results are consistent with our notion that rats with HPC lesions tend to segregate environmental stimuli and condition responses equally to each component. That is, they prefer associative, rather than relational, context in learning new responses. Normal animals, however, link the CS and the context into a relational representation.

Since it is reliably established that rats with HPC lesions can condition to (associative) context when the context is highly salient, it would be desirable to use this paradigm to test RA of a fear response to a CS and context in a between-groups design. We predict a temporally graded RA in HPC rats in both tests on the argument that a conditioned fear response (to the CS or context) would be context-dependent in its early stages and vulnerable to effects of HPC damage. With the passage of time, either response would become increasingly free of relational context and presumably less susceptible to the effects of HPC lesions. Further, a follow-up experiment in which context is manipulated at critical times, similar to that proposed for the food preference paradigm, would provide additional assessment of the context-free/context-dependent hypothesis.

In contrast to the above tasks, tests of spatial memory are uniquely relationally- and context-dependent in that a particular spatial location must be learned and remembered in relation to a background of environmental cues that defines the location. When those cues are removed, as by turning off the lights or by changing their relationship to the particular location, the location loses its spatial relational context and can be found only if the animal can effectively use landmarks or response-based cues. Rats or mice with HPC lesions are generally impaired in forming spatial memories in a variety of tasks (e.g. radial arm maze, Morris water maze, blind-alley mazes). Only a few studies have investigated the effects of HPC lesions on pre-operatively acquired spatial memories and usually in the Morris water maze (e.g. [3,6,45,61]). Nevertheless, the results consistently point to a severe impairment that extends backwards in time with a flat gradient. As indicated above, in a recently completed study, we extended these results to a cross-shaped maze.

To our knowledge, there are no reports that animals with HPC lesions exhibit temporally graded RA for spatial memory as seen in amnesic patients with MTL damage. However, it must be emphasized that spatial memory was retained in these patients, if only for the general features of extremely well-learned spatial environments $[62,80]$. That is, patients recalled gist-like information for neighbourhoods in which they lived for long periods of time and which they experienced in different ways and from different perspectives. Given the nature of spatial memory and its unique relation to the HPC, this type of varied experience may be necessary to enable the formation of a representation that will allow the abstraction of general features of a spatial environment that can be remembered independently of detailed elements. These conditions contrast with those in the animal studies where training typically consists of a limited number of invariant trials in an environment where there are only a limited number of spatial cues.

No studies have attempted to emulate in animals the human experience of forming complex representations 


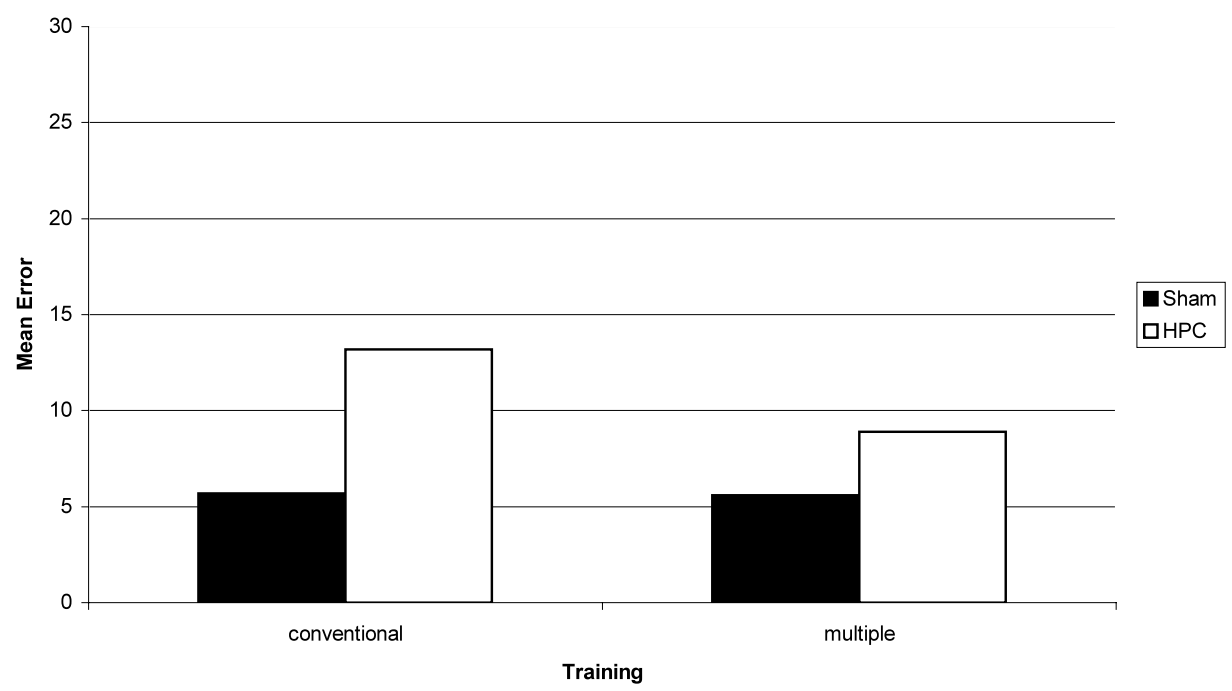

Fig. 3. The influence of over-training on maze performance following hippocampal lesions in rats. Values represent group mean error.

of an extremely familiar spatial environment. However, a recent study in our lab [55] may provide a first step in this direction. In this study, rats were trained pre-operatively on a complex blind alley maze under two conditions. In one, designed to promote the formation of multiple representations of the spatial environment, the maze was positioned in different locations with the goal box remaining in a constant location relative to the surrounding environment. Local, intra-maze cues were made highly salient, and training trials were administered under dim or normal illumination. Another group of rats received the same amount of training with the maze in a fixed position, under invariant conditions, and without salient intra-maze cues.

Following HPC or control surgery, rats were tested in the same maze without intra-maze cues. In a dramatic result, HPC rats trained in the multiple-representation condition performed significantly better than HPC rats trained in the conventional manner. No such advantage was conferred on the control rats (see Fig. $3)$. This finding indicates that training from different perspectives supports the development of a highly flexible spatial representation that permits rats with HPC lesions to relate the spatial layout of a maze to the external environment. To test the robustness of this effect, the same rats were tested on the familiar maze in a different environment. Unable to use the established spatial representations, the multiple-representation HPC group did not maintain its advantage over the conventionally trained HPC group, and their error scores did not differ.

At this point, these results must be considered preliminary, but they do suggest that elaborate encoding of a spatial environment will contribute to representations of the environment that will support memory, at least in its general features. It is important to note that in the multiple-representation training condition, HPC groups performed worse than controls that were trained in the same way. This indicates that not all the relevant spatial information was retained after HPC lesions.

The results of this experiment point to a close correspondence between memory for well-learned spatial environments in HPC-damaged animals and humans. To reinforce this link, an experiment is needed in which rats are allowed to live in a 'complex' environment that is analogous to a 'neighbourhood' and see if memory of the environment can be retained post-operatively in a temporally graded manner. Indeed, such an experiment is underway in our lab. Our position predicts that, in line with observations of MTL amnesics, memory for 'neighbourhoods' experienced just before surgery will be vulnerable to effects of HPC lesions because of the reliance on relational context in forming and recovering the memory traces. However, memories formed much earlier will have had the opportunity to become additionally represented in terms of their general or gist-like features that relate to associative contextual cues. The component of the spatial memory that is represented in this way can be recovered by associative cues. Thus, after the passage of time, memories of the neighbourhood will be represented in terms of relational and associative contexts. We predict that, post-operatively, HPC groups, exposed to the neighbourhoods as young adults, will perform better than HPC groups exposed more recently to the neighbourhoods. The former groups will have access to memories based on associative context, whereas the latter will have to contend with inaccessable memories that are based on relational context. If our predictions are confirmed, they will help to reconcile the animal and human data and provide additional evidence that the distinction between different types of contextual information is crucial for under- 
standing recovery of spatial memory following HPC damage.

\section{Summary and conclusions}

A re-examination of the early literature on retrograde memory loss as well as consideration of new data have forced a re-evaluation of the standard consolidation hypothesis and promotion of an alternative theoretical model. With regard to human memory, the duration of RA sometimes is short, but more often RA for details of autobiographical events after large MTL lesions can extend for decades, or even a lifetime, far longer than would be biologically plausible for a consolidation process to last. RA for public events and personalities, however, is less extensive and often is temporally graded; this is truer still of semantic memory, which includes knowledge of new vocabulary and facts about the world and ourselves (i.e. our address, the names of friends, our job), what some have called personal semantics to distinguish them from autobiographical episodes $[7,26]$.

The distinction between temporally extensive and temporally limited RA also applies to spatial memory, and a pattern of preserved and impaired function resembles that for other types of knowledge. Patients with bilateral MTL damage can retain schematic cognitive maps of old neighbourhoods that are adequate for navigation but which lack topographical details and local environmental features, such as the appearance and location of particular homes, that would allow the individual to retain detailed cognitive maps of their locale [62]. In this way, the pattern of preserved and impaired spatial memory is akin to that observed for episodic and semantic memories-the gist is retained but the details are lost.

In view of the limitations of consolidation theory, Nadel and Moscovitch [48-50] concluded that the HPC is not a temporally limited memory system, at least as far as certain types of information are concerned. They proposed that the MTL system is needed to represent even old memories in rich, multifaceted details, be they autobiographical or spatial, for as long as the memories exist, even if they are decades old. Neocortical structures, on the other hand, are sufficient to form domainspecific and semantic representations based on regularities extracted from repeated experiences for words, objects, people and environments, or, in other words, as context-free memories in the sense that we use the term in this paper. Even autobiographical episodes fall into this category when repeated recollections allow for the abstraction of a gist of the episode, or the core elements without contextual detail. The MTL system may aid in the initial formation of these context-free neocortical representations because, ini- tially, aspects of them were context-dependent. However, once formed and free of relational context, they can exist on their own.

These ideas about the role of the HPC and neocortex in retention and retrieval of old memories are embodied in the Multiple Trace Theory (MTT) proposed by Nadel and Moscovitch [48,49] to account for the evidence on RA. According to MTT, the memory trace of an episode or contextually rich event consists of a bound ensemble of neocortical and MTL/HPC neurons. Formation and consolidation of these traces, or cohesion [43], is relatively rapid, lasting on the order of seconds or, at most, days. Each time an old memory is retrieved, a new HPC-mediated trace is created so that old memories are represented by more or stronger traces than are new ones, making them more resistant to partial lesions of the MTL. This proliferation of traces renders older memories less susceptible to disruption from brain damage than more recent ones. Thus, in regard to the formation of new traces and their rapid short-term consolidation, MTT resembles standard consolidation theories. It differs from the standard theory in that MTT does not posit an additional longterm consolidation process but gradually makes memory for contextually rich events less dependent on HPC and related structures and more dependent on the neocortex.

MTT provides a way of understanding the patterns of RA reported after damage to the HPC complex. With regard to rich, context-dependent memory, the extent and severity of RA and perhaps the slope of the gradient are related to the amount of damage to the extended HPC complex, which includes the HPC and adjacent structures in MTL and neocortex. In humans, long-lasting HPC dependence applies primarily to autobiographical memory and perhaps to detailed memories of public events, personalities, and places. In rats, it applies to spatial memory and other types that are shown to be context-dependent.

In contrast, remote memories that are less contextually rich, or even context-free, are not similarly dependent on the continuing function of the HPC complex. Thus, in humans, memories for the gist of events, for familiarity of names, and faces, as well as for personal and lexical semantics can survive HPC damage after some relatively short time has elapsed between acquisition and lesion onset. In fact, such memories may be acquired even with extensive HPC damage, as VarghaKhadem et al. [86] have shown in children with early HPC lesions.

A major objective of this paper was to show that there is a correspondence between the animal and human literatures on HPC involvement in remote memory and that both sets of data can be interpreted within a common theoretical framework. To achieve this, it was necessary to draw analogies, on the one hand, between 
episodic memory in humans and memory that is tied to relational context in animals, and, on the other hand, between semantic memory in humans and memory in animals that is free of relational context. We proposed that the latter memories are tied to non-relational, associative context.

Consistent with this view, the evidence shows that rats with HPC lesions exhibit extensive RA for memories that are tied to relational context, as seen most reliably in tests of spatial memory. Conversely, HPC rats show only a brief, temporally graded RA for context-free memories such as are needed for establishing familiarity with objects and for taste preferences after HPC lesions, and may even acquire these memories normally without the HPC. In humans, rats, and presumably other mammals, neocortex and, perhaps, non-HPC structures in MTL, are sufficient to create context-free memories at acquisition with minimal or short-term HPC support. These structures extract regularities from repeated experiences with object, words, and other material, thereby freeing them from the relational context of which they were initially a part. The process of forming context-free (semantic) representations may initially require some HPC support, which would account for the temporally graded, relatively brief RA for context-free memory as seen in contextual fear conditioning, object discrimination, and food-preference tasks.

In this paper, we focussed primarily on the role of the HPC in remote memory. At the same time, we made certain assumptions about the involvement of extra-HPC structures and drew on evidence that extraHPC structures within the MTL region are crucial for context-free memories. More research is needed to determine the specific contributions of the various nonHPC structures and to show how they interact with neocortex and other brain areas to form and retain context-free memories. As a guiding principle of this research, it is our view that the respective functions of these structures, as they are identified, will be relevant to the acquisition of specific types of information (e.g. objects, context-free information) as well as the longterm retention of that information.

\section{Acknowledgements}

Research reported in this paper and the preparation of this manuscript were supported by a Natural Sciences and Engineering Research Council (NSERC) grant to GW, a Canadian Institutes of Health Research (CIHR) grant to GW and MM, and a NSERC postgraduate scholarship and CIHR doctoral award to RSR. The authors wish to thank Doug Caruana and Kimberly Oxbro for their technical assistance.

\section{References}

[1] Anagnosteras SG, Maren S, Fanselow MS. Temporally graded retrograde amnesia of contextual fear after hippocampal damage in rats: within-subjects examination. J Neurosci 1999; 10:3295-301.

[2] Albert MS, Butters N, Levin J. Temporal gradients in the retrograde amnesia of patients with alcoholic Korsakoff's disease. Arch Neurol 1979;36:211-6.

[3] Astur RS, Mumby DG, Weisend MP, Sutherland RJ. Hippocampal damage in rats causes retrograde amnesia for place navigation but not object discriminations. Soc Neurosci Abstr 1994;20:1015.

[4] Beatty WW, Salmon DP, Berstein N, Butters N. Remote memory in a patient with amnesia due to hypoxia. Psychol Med 1987; 17:657-65.

[5] Beatty WW, Salmon DP, Butters N, Heindel WC, Granholm EA. Retrograde amnesia in patients with Alzheimer's disease or Huntington's disease. Neurobiol Aging 1988;9:181-6.

[6] Bolhuis JJ, Stewart CA, Forest EM. Retrograde amnesia and memory reactivation in rats with ibotenate lesions to the hippocampus or subiculum. Q J Exp Psychol 1994;47:129-50.

[7] Cermak LS, O'Connor M. The anterograde and retrograde retrieval ability of a patient with amnesia due to encephalitis. Neuropsychologia 1983;21:213-34.

[8] Cho YH, Kesner RP, Brodale S. Retrograde and anterograde amnesia for spatial discrimination in rats: role of hippocampus, entorhinal cortex, and parietal cortex. Psychobiology 1995;23:185-94.

[9] Cipolotti L, Shallice T, Chan D, Fox N, Scahill R, Harrison G, Stevens J, Rudge P. Long-term retrograde amnesia...the crucial role of the hippocampus. Neuropsychologia 2001;39:151-72.

[10] Cohen NJ, Squire LR. Retrograde amnesia and remote memory impairment. Neuropsychologia 1981;19:337-56.

[11] Conway MA, Turk DJ, Miller SL, Logan J, Nebes RD, Meltzer CC, Becker JT. A positron emission tomography (PET) study of autobiographical memory retrieval. Memory 1999;56:679-702.

[12] Crovitz HF, Schiffman H. Frequency of episodic memories as a function of their age. Bull Psychonomic Soc 1974;4:519-21.

[13] Eichenbaum H. The hippocampus and mechanisms of declarative memory. Behav Brain Res 1999;103:123-33.

[14] Eichenbaum H. Hippocampus: mapping or memory? Curr Biol 2000;10:R785-7.

[15] Ellen P, Deloache P. Hippocampal lesions and spontaneous alternation behavior in the rat. Physiol Behav 1973;3:857-60.

[16] Fink GR, Markowitsch HJ, Reinkemeier M, Bruckbauer T, Kessler J, Heiss WD. Cerebral representation of one's own past: neural networks involved in autobiographical memory. $\mathbf{J}$ Neurosci 1996;16:4275-82.

[17] Fujii T, Moscovitch M, Nadel L. Memory consolidation, retrograde amnesia, and the temporal lobe. In: Cermak L, editor. Handbook of Neuropsychology, vol. 2. Amsterdam: Elsevier, 2000:223-50.

[18] Gabrieli JD, Cohen NJ, Corkin S. The impaired learning of semantic knowledge following bilateral medial temporal-lobe resection. Brain Cogn 1988;7:157-77.

[19] Galef BG Jr, Wigmore SW, Kennett DJ. A failure to find socially mediated taste aversion learning in Norway rats $(R$. norvegicus). J Comp Psychol 1983;97:358-63.

[20] Good M, Honey RC. Conditioning and contextual retrieval in hippocampal rats. Behav Neurosci 1991;105:499-509.

[21] Hamann SB, Squire LR. On the acquisition of new declarative knowledge in amnesia. Behav Neurosci 1995;109:1027-44.

[22] Holdstock JS, Mayes AR, Cezayirli E, Isaac CL, Aggleton JP, Roberts N. A comparison of egocentric and allocentric spatial 
memory in a patient with selective hippocampal damage. Neuropsychologia 2000;38:410-25.

[23] Kim JJ, Clark RE, Thompson RF. Hippocampectomy impairs the memory of recently, but not remotely, acquired trace eyeblink conditioned response. Behav Neurosci 1995;109:195-203.

[24] Kim JJ, Fanselow MS. Modality-specific retrograde amnesia of fear. Science 1992;256:675-7.

[25] Kinsbourne M, Wood F. Short-term memory processes and the amnesic syndrome. In: Deutsch D, Deutsch JA, editors. ShortTerm Memory Processes. New York: Academic Press, 1975:258-91.

[26] Kopelman MD, Wilson BA, Baddeley AD. The autobiographical memory interview: a new assessment of autobiographical and personal semantic memory in amnesic patients. J Clin Exp Neuropsychol 1989;5:724-44.

[27] Lewis DJ, Misanin JR, Miller RR. Recovery of memory following amnesia. Nature 1968;220:704-5.

[28] MacKinnon D, Squire LR. Autobiographical memory in amnesia. Psychobiology 1989;17:247-56.

[29] Maguire EA. Neuroimaging studies of autobiographical event memory. Philos. Trans. R. Soc. London B Biol. Sci., 2001, in press.

[30] Maguire EA, Frackowiak RSJ, Frith CD. Learning to find your way: A role for the human hippocampal formation. Proc R Soc London B Biol Sci 1996;263:1745-50.

[31] Maguire EA, Frackowiak RSJ, Frith CD. Recalling routes around London: Activation of the right hippocampus in taxi drivers. J Neurosci 1997;17:7103-10.

[32] Maguire EA, Mummery CJ, Buchel C. Patterns of hippocampal-cortical interaction dissociate temporal lobe memory subsystems. Hippocampus 2000;10:475-82.

[33] Maguire EA, Mummery CJ. Differential modulation of a common memory retrieval network revealed by positron emission tomography. Hippocampus 1999;9:54-61.

[34] Maguire EA, Vargha-Khadem F, Mishkin M. The effects of bilateral hippocampal damage on fMRI regional activations and interactions during memory retrieval. Brain 2001;124:1156-70.

[35] Marr D. Simple memory: a theory for archicortex. Philos Trans R Soc London Biol Sci 1971;262:23-81.

[36] Marslen-Wilson WD, Teuber HL. Memory for remote events in anterograde amnesia: recognition of public figures from news photographs. Neuropsychologia 1975;13:353-64.

[37] McClelland JL, McNaughton BL, O'Reilly RC. Why there are complementary learning systems in the hippocampus and neocortex: Insights from the successes and failures of connectionist models of learning and memory. Psychol Rev 1995; 102:419-57.

[38] Miller RR, Springer AD. Amnesia, consolidation, and rertrieval. Psychol Rev 1973;80:69-79.

[39] Milner B, Corkin S, Teuber HL. Further analysis of the hippocampal amnesic syndrome: 14-year follow-up of H.M. Neuropsychologia 1968;6:215-34.

[40] Milner B, Squire LR, Kandel ER. Cognitive neuroscience and the study of memory. Neuron 1998;20:445-68.

[41] Morris RGM, Garrud P, Rawlins JNP, O'Keefe J. Place navigation impaired in rats with hippocampal lesions. Nature 1982;297:681-3.

[42] Moscovitch M. Multiple dissociations of function in amnesia. In: Cermak LS, editor. Human Memory and Amnesia. Hillsdale, NJ: Erlbaum, 1982:337-70.

[43] Moscovitch M. Recovered consciousness: a hypothesis concerning modularity and episodic memory. J Clin Exp Neuropsychol 1995; 17:276-90.

[44] Moscovitch M, Nadel L. Consolidation and the hippocampal complex revisited: In defense of the multiple-trace model. Curr Opin Neurobiol 1998;8:297-300.
[45] Mumby DG, Astur RS, Weisend MP, Sutherland RJ. Retrograde amnesia and selective damage to the hippocampal formation: memory for places and object discriminations. Behav Brain Res 1999;106:97-107.

[46] Murray EA, Bussey TJ. Consolidation and the medial temporal lobe revisited: methodological considerations. Hippocampus 2001;11:1-7.

[47] Nadel L, MacDonald L. Hippocampus: Cognitive map or working memory? Behav Neural Biol 1980;29:405-9.

[48] Nadel L, Moscovitch M. Memory consolidation, retrograde amnesia and the hippocampal complex. Curr Opin Neurobiol 1997; 7:217-27.

[49] Nadel L, Moscovitch M. The hippocampal complex and longterm memory revisited. Trends Cogn Sci 2001;5:228-30.

[50] Nadel L, Samsonovich A, Ryan L, Moscovitch M. Multiple trace theory of human memory: computational, neuroimaging and neuropsychological results. Hippocampus 2000;10:352-68.

[51] Nunn JA, Wilkinson ID, Spencer T, Khiami R, Griffiths PD, Mayes A. Both long and short-term topographical memory recall produces activation of the medial temporal lobe: A functional magnetic resonance study, Proc. Int. Soc. Mag. Reson. Med., 2000; in press.

[52] O'Keefe J, Dostrovsky J. The hippocampus as a spatial map: preliminary evidence from unit activity in the freely-moving rat. Brain Res 1971;34:171-5.

[53] O'Keefe J, Nadel L. The Hippocampus as a Cognitive Map. Oxford: Clarendon Press, 1978.

[54] Olton DS, Becker JT, Handelman GH. Hippocampus, space and memory. Behav Brain Sci 1979;2:313-65.

[55] Oxbro K. Hippocampal lesions and multiple representations of spatial memory: effects of difference exposures to the spatial environment. Honours Thesis, Trent University 2001.

[56] Paller KA. Neural measures of conscious and unconscious memory. Behav Neurol 2000;12:127-41.

[57] Penfield W, Mathieson G. Memory: autopsy findings and comments on the role of the hippocampus in experiential recall. Arch Neurol 1974;31:145-54.

[58] Penfield W, Milner B. Memory deficit produced by bilateral lesions in the hippocampal zone. Arch Neurol Psychiatry 1958;79:475-97.

[59] Penick S, Solomon R. Hippocampus, context and conditioning. Behav Neurosci 1991;105:611-7.

[60] Reed JM, Squire LR. Retrograde amnesia for facts and events: Findings from four new cases. J Neurosci 1998;18:3943-54.

[61] Riedel G, Micheau J, Lam AG, Roloff Ev, Martin SJ, Bridge J, Hoz Ld, Poeschel B, McCulloch J, Morris RG. Reversible neural inactivation reveals hippocampal participation in several memory processes. Nat Neurosci 1999;2:898-905.

[62] Rosenbaum RS, Priselac S, Kohler S, Black SE, Gao F, Nadel L, Moscovitch M. Remote spatial memory in an amnesic person with extensive bilateral hippocampal lesions. Nat Neurosci 2000;3:1044-8.

[63] Ryan L, Nadel L, Keil T, Putnam K, Schnyer D, Trouard T, Moscovitch M. The hippocampal complex and retrieval of recent and very remote autobiographical memories: evidence from functional magnetic resonance imaging in neurologically intact people. Hippocampus, in press.

[64] Salmon DP, Zola-Morgan S, Squire LR. Retrograde amnesia following combined hippocampus-amygdala lesions in monkeys. Psychobiology 1987;15:37-47.

[65] Schacter DL, Chiu CYP, Ochsner KN. Implicit memory: a selective review. Annu Rev Neurosci 1993;16:159-82.

[66] Scoville WB, Milner B. Loss of recent memory after bilateral hippocampal lesions. J Neurol Neurosurg Psychiatry 1957;20:11-21. 
[67] Smith ML, Milner B. The role of the right hippocampus in the recall of spatial location. Neuropsychologia 1981;19:781-93.

[68] Squire LR. On the course of forgetting in very long-term memory. J Exp Psychol Learn Mem Cogn 1989;15:241-5.

[69] Squire LR. Memory and the hippocampus: A synthesis from findings with rats, monkeys and humans. Psychol Rev 1992;99:195-231.

[70] Squire LR, Alvarez P. Retrograde amnesia and memory consolidation: a neurobiological perspective. Curr Opin Neurobiol 1995;5:169-77.

[71] Squire LR, Cohen NJ, Nadel L. The medial temporal region and memory consolidation: a new hypothesis. In: Weingartner H, Parker E, editors. Memory Consolidation. Hillsdale, NJ: Erlbaum, 1984:185-210.

[72] Squire LR, Haist F, Shimamura AP. The neurology of memory: Quantitative assessment of retrograde amnesia in two groups of amnesic patients. J Neurosci 1989;9:828-39.

[73] Squire LR, Slater PC, Chace PM. Retrograde amnesia: temporal gradient in very long-term memory following electroconvulsive therapy. Science 1975;187:77-9.

[74] Squire LR, Zola-Morgan S. Structure and function of declarative and nondeclarative memory systems. Proc Natl Acad Sci USA 1996;93:13515-22.

[75] Squire LR, Zola-Morgan S. Amnesia, memory and brain systems. Philos Trans R Soc London 1997;352:1663-73.

[76] Squire LR, Zola SM. Episodic memory, semantic memory, and amnesia. Hippocampus 1998;8:205-11.

[77] Sutherland RJ, Arnold KA, Rodriguez AR. Anterograde and retrograde effects on place memory after limbic or diencephalic damage. Soc Neurosci Abstr 1987;13:1066.

[78] Sutherland RJ, McDonald RJ. Hippocampus, amygdala, and memory deficits in rats. Behav Brain Res 1990;37:57-79.

[79] Sutherland RJ, Whishaw IQ, Kolb B. A behavioural analysis of spatial localization following electrolytic, kainate-or colchicineinduced damage to the hippocampal formation in the rat. Behav Brain Res 1983;7:133-53.

[80] Teng E, Squire LR. Memory for places learned long ago is intact after hippocampal damage. Nature 1999;400:675-7.

[81] Teyler TJ, DiScenna P. The hippocampal memory indexing theory. Behav Neurosci 1986;100:147-54.

[82] Tolman EC. Cognitive maps in rats and man. Psychol Rev 1948;55:189-208.

[83] Tulving E. Episodic and semantic memory. In: Tulving E, Donalson W, editors. Organization of Memory. New York: Academic Press, 1972:381-403.

[84] Tulving E, Schacter DL, McLachlan DR, Moscovitch M. Priming of semantic autobiographical knowledge: A case study of retrograde amnesia. Brain Cog 1988;8:3-20.

[85] Van der Linden M, de Partz M, Schils J, Seron X. Semantic and autobiographical memory: Neuropsychological dissocia- tions? In: Conway MA, Rubin DC, Spinnler H, editors. Theoretical Perspectives on Autobiographical Memory. Boston: Kluwer Academic Publishers, 1992:473-94.

[86] Vargha-Khadem F, Gadian DG, Watkins KE, Connelly A, Van Paesschen W, Mishkin M. Differential effects of early hippocampal pathology on episodic and semantic memory. Science 1997;277:376-80.

[87] Verfaellie M, Reiss L, Roth HL. Knowledge of New English vocabulary in amnesia: an examination of premorbidly acquired semantic memory. J Int Neuropsychol Soc 1995;1:44353.

[88] Viskontas IV, McAndrews MP, Moscovtich M. Remote episodic memory deficits in patients with unilateral temporal lobe epilepsy and excisions. J Neurosci 2000;20:5853-7.

[89] Warrington EK, McCarthy RA. The fractionation of retrograde amnesia. Brain Cogn 1988;7:184-200.

[90] Winocur G. Radial-arm-maze behavior by rats with dorsal hippocampal lesions: effect of cuing. J Comp Physiol Psychol 1982;96:155-69.

[91] Winocur G. Anterograde and retrograde amnesia in rats with dorsal hippocampal or dorsomedial thalamic lesions. Behav Brain Res 1990;38:145-54.

[92] Winocur G. Hippocampal lesions alter conditioning to conditional and context stimuli. Behav Brain Res 1997;88:219-29.

[93] Winocur G, Bindra D. Effects of additional cues on passive avoidance learning and extinction in rats with hippocampal lesions. Physiol Behav 1976;17:915-20.

[94] Winocur G, Black AH. Cue-induced recall of a passive avoidance response by rats with hippocampal lesions. Physiol Behav 1978;21:39-44.

[95] Winocur G, Breckenridge CB. Cue-dependent behavior of hippocampally damaged rats in a complex maze. J Comp Physiol Psychol 1973;82:512-22.

[96] Winocur G, McDonald RJ, Moscovitch M. Anterograde and retrograde amnesia in rats with large hippocampal lesions. Hippocampus 2001;11:18-26.

[97] Winocur G, Moscovitch M. Anterograde and retrograde amnesia after lesions to frontal cortex in rats. $\mathbf{J}$ Neurosci 1999;19:9611-7.

[98] Winocur G, Rawlins JNP, Gray JA. The hippocampus and conditioning to contextual cues. Behav Neurosci 1987;5:61725.

[99] Zola-Morgan S, Squire LR. The primate hippocampal formation: evidence for a time-limited role in memory storage. Science 1990;250:288-90.

[100] Zola-Morgan S, Squire LR, Amaral DG. Human amnesia and the medial temporal region: enduring memory impairment following a bilateral lesion limited to field CA1 of the hippocampus. J Neurosci 1986;6:2950-67. 